\title{
Importance des cultes dans la préservation des espèces d'arbre, le cas du samba (Triplochiton scleroxylon K. Schum.) au Bénin
}

Gabin GANKA'

Kolawolé Valère SALAKO ${ }^{2}$

Adandé Belarmain FANDOHAN ${ }^{1,2}$

${ }^{1}$ Université nationale d'agriculture École de foresterie tropicale Laboratoire des sciences végétales, horticoles et forestières

Unité de recherche en foresterie et conservation des bioressources BP 43, Kétou

Bénin

\section{${ }^{2}$ Université d'Abomey-Calavi} Faculté des sciences agronomiques Laboratoire de biomathématiques et d'estimations forestières 04 BP 1525, Cotonou Bénin

\section{Auteur correspondant /} Corresponding author:

Gabin GANKA - gabinganka@gmail.com

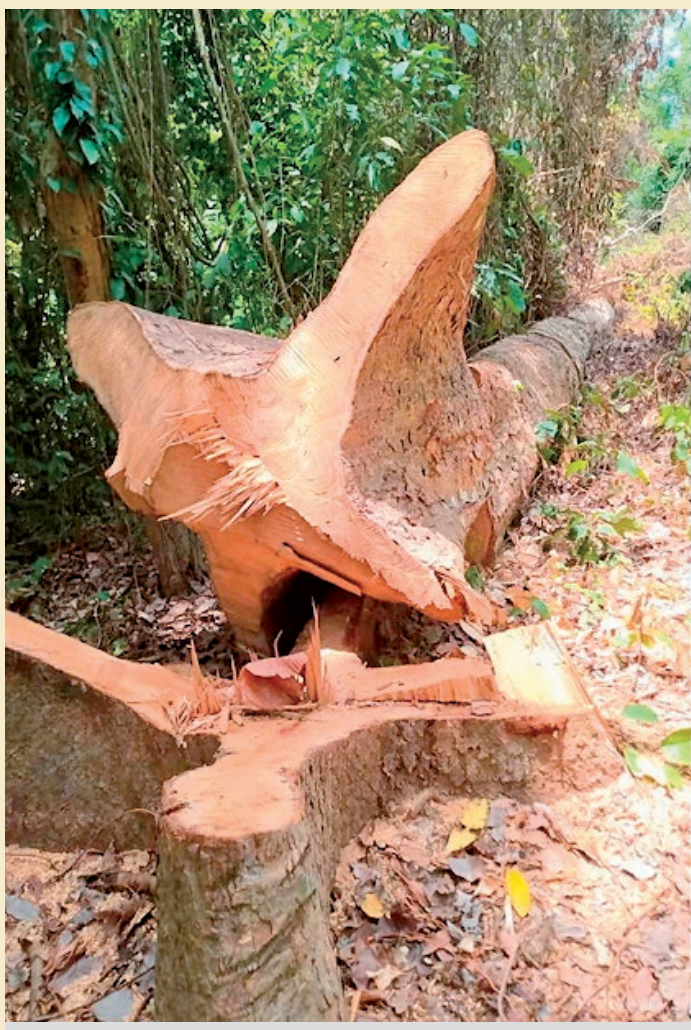

Photos 1.

Triplochiton scleroxylon en forêt sacrée dans la région guinéo-congolaise du Bénin. Triplochiton scleroxylon in the sacred forest in the Guinean-Congolese region of Benin. Photos G. Ganka.

Doi : 10.19182/bft2022.351.a36866 - Droit d'auteur ๔ 2022, Bois et Forêts des Tropiques - @ Cirad - Date de soumission : 2 mars 2021 ;

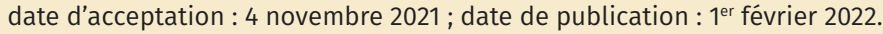

\section{Ccirad (a)}

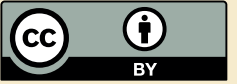

Citer l'article / To cite the article

Ganka G., Salako K. V., Fandohan A. B., 2022. Importance des cultes dans la préservation des espèces d'arbre, le cas du samba (Triplochiton scleroxylon K. Schum.) au Bénin. Bois et Forêts des Tropiques, 351:53-65. Doi : https:// doi.org/10.19182/bft2022.351.a36866 


\section{RÉSUMÉ}

Importance des cultes dans

la préservation des espèces d'arbre, le cas du samba (Triplochiton scleroxylon K. Schum.) au Bénin

Triplochiton scleroxylon K. Schum. est l'une des espèces vénérées des écosystèmes sacrés du Bénin qui revêt une importance socioculturelle. Toutefois, les peuplements naturels de l'espèce sont en déclin et les informations sur ses usages sont quasi non documentées. Cette étude évalue les connaissances locales sur les usages de l'espèce et la distribution desdites connaissances entre groupes ethnolinguistiques, culturels et cultuels au Bénin. Des enquêtes ethnobotaniques individuelles ont été réalisées auprès de 411 adeptes des divinités liées à la conservation de l'espèce au Bénin. Les indices ethnobotaniques tels que la fréquence relative de citation (FRC), la valeur d'usage (VU) total et par organe, et l'indice d'importance culturelle (IC) ont été utilisés pour quantifier la popularité, l'utilisation et l'importance socioculturelle de T. scleroxylon, respectivement. Un modèle linéaire généralisé avec la distribution de la binomiale négative a été réalisé pour examiner la variation de la VU entre groupes ethnolinguistiques, culturels et cultuels. Au total, 36 usages spécifiques catégorisés en neuf types d'usages ont été rapportés. Les usages cultuels, médicinaux, artisanaux et comme bois d'œuvre sont les plus cités. Toutes les parties de la plante sont prisées dans la tradition du culte Oro. Les feuilles, racines et écorces sont les organes utilisés pour traiter diverses affections dont les plus populaires sont les affections digestives et gynécologiques. Les connaissances et les usages de l'espèce varient significativement entre les groupes ethnolinguistiques et cultuels. Une singularité des connaissances de T. scleroxylon a été observée chez les adeptes du culte Oro. La disparition de cette espèce pourrait avoir des conséquences majeures sur l'identité et la stabilité culturelle et cultuelle des peuples pratiquant cette tradition. Les usages médicinaux populaires rapportés offrent aussi des perspectives pour la valorisation médicinale de l'espèce.
ABSTRACT

On the importance of the sacred in the preservation of tree species: the case of Samba (Triplochiton scleroxylon K. Schum.) in Benin

Triplochiton scleroxylon K. Schum. is a species of socio-cultural importance as one of the trees that are venerated in the sacred ecosystems of Benin. However, natural stands of the species are declining and virtually no detailed information on its local uses has been documented. The aim of this study was to assess local knowledge on uses of the species and its distribution among ethnolinguistic, cultural and faith groups in Benin. Individual ethnobotanical interviews were conducted with 411 believers in deities associated with conservation of this tree species. Ethnobotanical indices such as relative frequency of citation, use value (total and per tree organ), and the cultural importance index were used to quantify the popularity, uses and socio-cultural importance of $T$. scleroxylon. A negative binomial generalized linear model was used to examine the variations in use value between ethno-linguistic, cultural and faith groups. A total of 36 specific uses classified into nine use types were reported. Cultural, medicinal, food, artisanal and timber uses were the most popular. All parts of the plant are valued in the tradition of Oro worship. Leaves, roots and bark are the organs of $T$. scleroxylon used to treat various ailments, the most common being digestive and gynaecological. Knowledge and uses of the species vary significantly among the groups surveyed. We found singular knowledge, uses and importance of $T$. scleroxylon among followers of the Oro cult. The disappearance of this species could have major consequences for the identity and the cultural and religious stability of the people practising this tradition. The popular medicinal uses reported also offer prospects for promoting the medicinal properties of the species.

Keywords: Triplochiton scleroxylon, Samba, traditional knowledge, use value, cultural importance, religious importance, sacred forests, Benin.

\section{RESUMEN}

\author{
Importancia de los cultos en \\ la conservación de las especies arbóreas, \\ el caso de la samba (Triplochiton \\ scleroxylon K. Schum.) en Benín
}

El Triplochiton scleroxylon K. Schum. es una de las especies veneradas de los ecosistemas sagrados de Benín, con una gran importancia sociocultural. Sin embargo, las masas forestales naturales de la especie están disminuyendo y la información sobre sus usos está casi sin documentar. Este estudio evalúa los conocimientos locales sobre los usos de la especie y la distribución de dichos conocimientos entre los grupos etnolingüísticos, culturales y cultuales en Benín. Se realizaron encuestas etnobotánicas individuales entre 411 devotos de deidades vinculadas a la conservación de la especie en Benín. Para cuantificar la popularidad, el uso y la importancia sociocultural de la T. scleroxylonse se utilizaron indices etnobotánicos como la frecuencia relativa de citación (FRC); el valor de uso (VU) total y por órgano, y el índice de importancia cultural (IC), respectivamente. Se realizó un modelo lineal generalizado con la distribución binomial negativa para examinar la variación del VU entre los grupos etnolingüísticos, culturales y cultuales. En total, se recogieron 36 usos específicos, clasificados en nueve tipos de uso. Los usos más citados son cultuales, medicinales, artesanales y como madera de construcción. Todas las partes de la planta se valoran en la tradición del culto Oro. Las hojas, las raíces y las cortezas son los órganos utilizados para tratar diversas afecciones, las más populares de las cuales son las digestivas y las ginecológicas. El conocimiento y uso de la especie varían significativamente entre los grupos etnolingüísticos y cultuales. Se ha observado una singularidad en el conocimiento de la T. scleroxylon entre los devotos del culto Oro. La desaparición de esta especie podría tener importantes consecuencias para la identidad y la estabilidad cultural y cultual de los pueblos que practican esta tradición. Los usos medicinales populares señalados también ofrecen perspectivas para la valorización medicinal de la especie.

Palabras clave: Triplochiton scleroxylon, samba, conocimientos tradicionales, valor de uso, importancia cultural, bosques sagrados, Benín.
Mots-clés : Triplochiton scleroxylon, samba, connaissances traditionnelles, valeur d'usage, importance culturelle, forêts sacrées, Bénin. 
Depuis les temps anciens, l'humanité dépend de différents produits des forêts pour les besoins quotidiens. Les forêts représentent une importante source de satisfaction des besoins élémentaires des populations, notamment s'alimenter et se soigner (Cavendish, 2000 ; Maxted et al., 2007 ; FAO, 2018). Les communautés résidant dans les villages et à proximité des forêts dépendent essentiellement des plantes et des produits végétaux pour leurs revenus et leurs moyens de subsistance car elles jouissent d'un droit spécifique sur les forêts et leurs produits (Rajesh et Nair, 2018). Les plantes ne sont pas seulement un moyen de subsistance ; les communautés locales sont aussi souvent engagées dans différentes pratiques magico-religieuses et rituelles où les plantes sont vénérées comme des dieux et déesses. La dépendance des sociétés, surtout rurales, vis-à-vis de ces ressources forestières peut constituer une menace pour l'équilibre des écosystèmes (Kalinganire et al., 2008 ; Fandohan et al., 2017). Prévenir la disparition de ces ressources nécessite de prendre en compte les considérations sociales dans les stratégies de conservation et de gestion durable des essences forestières.

En Afrique, les communautés rurales ont des traditions anciennes de collecte et d'utilisation de plusieurs espèces de plantes. Grâce à ces pratiques, ces communautés ont acquis des connaissances traditionnelles substantielles sur les propriétés des plantes et l'importance nutritionnelle, médicinale et spirituelle de la biodiversité locale (Johns et al., 1996). Bien que la modernisation ait été impliquée dans la réduction des connaissances et de l'utilisation de la biodiversité, ces savoirs endogènes ont été transmis de génération en génération (Pilgrim et al., 2008). Toutefois, de nombreuses études ont démontré la difficulté des générations plus âgées à transmettre de nos jours les connaissances traditionnelles à la jeune génération (Saynez-Vaquez et al., 2016) ; ce qui conduit à l'aliénation de cette génération quant à son environnement immédiat, et à la perte d'informations liées à la nature.

La recherche ethnobotanique prend en compte la relation entre les peuples et les plantes, y compris les croyances et les pratiques culturelles associées à diverses formes d'utilisation et de conservation de l'environnement naturel, conformément aux connaissances des communautés locales (Nolan et Turner, 2011). Ces connaissances permettent de comprendre les valeurs sociales, culturelles et économiques que ces communautés associent aux ressources autochtones avec lesquelles elles interagissent et partagent des liens séculaires. Elles sont donc devenues essentielles dans la biologie de la conservation (Pilgrim et al., 2008). Cependant, malgré la diversité des études ethnobotaniques sur plusieurs espèces autochtones d'Afrique au cours des deux dernières décennies, les informations sur plusieurs autres espèces de valeur restent éparses et non documentées, malgré leur statut d'espèce menacée dans certaines régions. C'est le cas du samba (Triplochiton scleroxylon K. Schum.) pour lequel les informations sur sa structure démographique et spatiale, son écophysiologie et sa sylviculture particulière restent encore fragmentaires.

Triplochiton scleroxylon, de la famille des Malvacées, est une espèce de l'étage dominant des forêts tropicales denses semi-décidues (Akoegninou et al., 2006). Troisième essence la plus exploitée en Afrique centrale pour la qualité de son bois (Bayol et al., 2012), T. scleroxylon fait également partie des premières plantes médicinales les plus utilisées au Nigeria pour guérir le diabète (Onoagbe et al., 1999). Au Bénin, elle est traditionnellement utilisée pour la fabrication d'objets d'art. Elle fait partie des essences les plus vénérées dans les couvents (Kokou et Sokpon, 2006). Arbre sacré du fétiche "Oro » chez les peuples Nagot au sud du Bénin (Palla et Louppe, 2002 ; Kokou et Sokpon, 2006 ; Adomou et al., 2011), son aire de distribution se réduit de plus en plus aux forêts sacrées d'Oro. Elle fait partie des centaines de plantes rituelles commercialisées sur les principaux marchés de plantes en Afrique de l'Ouest (Quiroz et van Andel, 2015). En raison des pouvoirs surnaturels qui sont souvent attribués aux arbres sacrés, leur récolte à des fins commerciales est devenue une entreprise rentable en Afrique (Quiroz et van Andel, 2015). Au Bénin, l'espèce fait face à de fortes menaces d'extinction (Adomou et al., 2011). Suivant les critères d'évaluation de la vulnérabilité des espèces de l'IUCN, elle fait partie des 105 espèces menacées du Bénin (Adomou et al., 2011). L'une des principales menaces sur l'espèce est la fragmentation des reliques de forêts denses semi-décidues qui constituent son habitat naturel. Son caractère sacré fait de $T$. scleroxylon une espèce inféodée à certains groupes ethnolinguistiques et cultuels dont les connaissances sur les usages de l'espèce, et l'importance culturelle, ne sont pas suffisamment développées.

La présente étude vise à évaluer les connaissances ethnobotaniques sur l'espèce et examiner la distribution de ces connaissances entre groupes ethnolinguistiques, culturels et cultuels au Bénin. En effet, plusieurs hypothèses ont été testées concernant la dynamique des connaissances des plantes. L'ensemble de ces hypothèses considère que le niveau de connaissance individuelle d'une plante est corrélé avec certains facteurs socioculturels et démographiques comme le sexe, l'âge, les us et coutumes (Sundriyal et al., 2004 ; Souto et Ticktin, 2012 ; Gaoué et al., 2017). Les femmes et les personnes âgées auraient ainsi tendance à avoir plus de connaissances sur les plantes médicinales; de même, les personnes avec un fort niveau d'alphabétisation ou ayant un meilleur accès à l'éducation formelle détiennent moins de connaissances sur l'usage des plantes (Voeks, 2007). Elles considèrent aussi que l'accumulation de connaissances sur les ressources naturelles, en particulier les plantes, est un processus dans le temps, de sorte qu'il est attendu que les personnes âgées aient plus de connaissances que les personnes jeunes (Hanazaki et al., 2013 ; Gaoué et al., 2017). 


\section{Matériel et méthode}

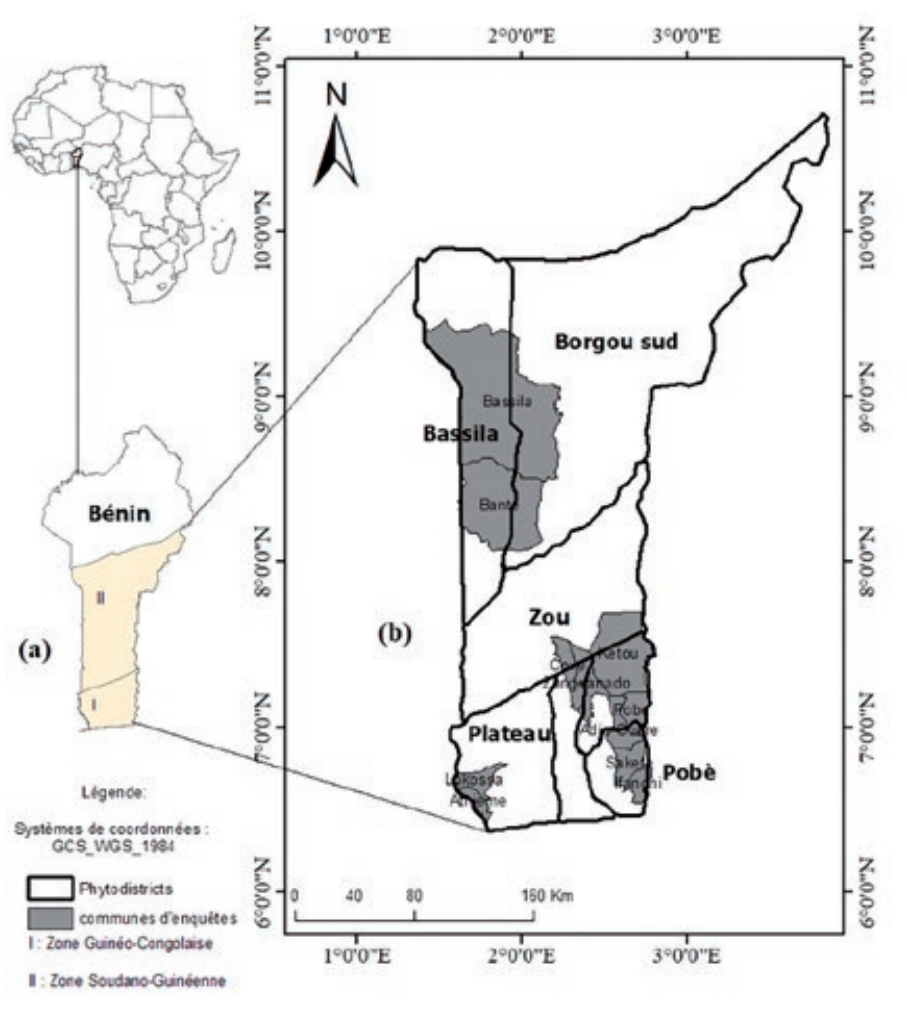

Figure 1.

Localisation géographique de la zone d'étude. Geographical location of the study area.

Plusieurs travaux ont testé l'objectivité de ces hypothèses. Cependant, le contexte dans lequel se fait la transmission des savoirs locaux peut influencer les résultats. Par exemple, les tabous et interdictions relatifs à certaines espèces sacrées dans certaines communautés en Afrique par rapport à la gente féminine ne permettent pas de vérifier ces hypothèses. Les hommes et les femmes peuvent posséder différents types de connaissances des usages des plantes en raison de leurs différents rôles définis culturellement. Le type de connaissance a de l'importance dans l'évaluation de la dynamique des connaissances sur l'usage des plantes (de Albuquerque et al., 2011 ; Souto et Ticktin, 2012 ; Gaoué et al., 2017). Il peut également être difficile de comparer les connaissances de toutes les plantes suivant l'âge car, dans les couvents, le niveau de responsabilité n'est pas forcement fonction de l'âge. Aussi la durée d'adhésion à un culte ou à une divinité peut-elle être un meilleur prédicteur de la dynamique des connaissances locales sur les plantes rituelles que l'âge. Il est donc clair que le degré des connaissances sur l'usage de T. scleroxylon pourrait varier indépendamment de l'âge, du sexe et du niveau d'instruction. Quels sont alors les facteurs prédictifs de la dynamique des connaissances sur l'usage traditionnel de T. scleroxylon, un arbre sacré et vénéré au Bénin?

\section{Modèle biologique d'étude}

Triplochiton scleroxylon est une espèce d'arbre de grande taille, à feuilles caduques, et ayant une faible densité de bois (320-440 $\mathrm{kg} / \mathrm{m}^{3}$ ) (Palla et Louppe, 2002), qui avec de grands contreforts à la base de son tronc peut dépasser $50 \mathrm{~m}$ de hauteur et $2 \mathrm{~m}$ de diamètre (Siepel et al., 2004). Elle fait partie des espèces principales de l'Afrique subsaharienne, dont l'aire de répartition s'étendait entre la Sierra Leone et le Congo (Fayolle et al., 2012). L'espèce est plus abondante sur les sols bien drainés, ferrugineux et fertiles, à une altitude inférieure à $500 \mathrm{~m}$. Les zones de son aire de répartition géographique ont un climat saisonnier avec des précipitations comprises entre 1100 et $1800 \mathrm{~mm}$ et une température oscillant entre 20 et $35^{\circ} \mathrm{C}$ (Hall et Bada, 1979). Au Bénin, il existe à ce jour peu d'évaluations quantitatives de l'abondance de l'espèce, de même que pour la structure de son peuplement; toutefois, sa principale zone d'occurrence au Bénin est l'espace guinéo-congolais, avec quelques individus dans la zone soudano-guinéenne, et principalement dans les forêts sacrées et quelques forêts classées (Akoegninou et al., 2006).

\section{Milieu d'étude}

La présente étude a été réalisée dans la zone de distribution de T. scleroxylon que sont le centre d'endémisme régional guinéo-congolais et la zone de transition régionale soudano-guinéenne au Bénin (figure 1). La région guinéo-congolaise du Bénin $\left(6^{\circ} 25^{\prime} \mathrm{N}\right.$ à $\left.7^{\circ} 30^{\prime} \mathrm{N}\right)$ représente la partie sud du pays. Le climat qui y règne est de type subéquatorial avec des précipitations annuelles moyennes de 900-1 $300 \mathrm{~mm}$. Le sol dominant dans cette région est de type ferrallitique profond, couvert par les forêts semidécidues (Adomou, 2005). Les groupes ethnolinguistiques dominants sont les Pédah, Idatcha, Nagot, Fon et Mahi.

La zone de transition soudano-guinéenne $\left(7^{\circ} 30^{\prime} \mathrm{N}-\right.$ $10^{\circ} 30^{\prime} \mathrm{N}$ ) représente la région méridionale du pays. Un climat subhumide y prévaut avec un régime à tendance unimodale de saison des pluies (1 000-1300 mm/an). Les sols ferrugineux couverts par les forêts sèches sont les types de sol dominants de cette zone. On y rencontre principalement les peuples Fon, Kotafon, Nagot/Yorouba, Goun, Wemé, Aïzo, Xwla, Tofin et Pédah (Adomou, 2005). Cependant, il est à noter que les forêts sacrées existent un peu partout au Bénin, dont l'ensemble représente une superficie non négligeable.

\section{Échantillonnage et collecte des données}

Une étude exploratoire a été conduite dans la zone de distribution de T. scleroxylon au Bénin (figure 1.a). Tous les villages à proximité des forêts sacrées abritant $T$. scleroxylon ont été retenus. Au total, 11 communes ont été considérées (figure 1.b). Cette première phase a permis d'identifier tous les couvents des cultes associés à la conservation des 


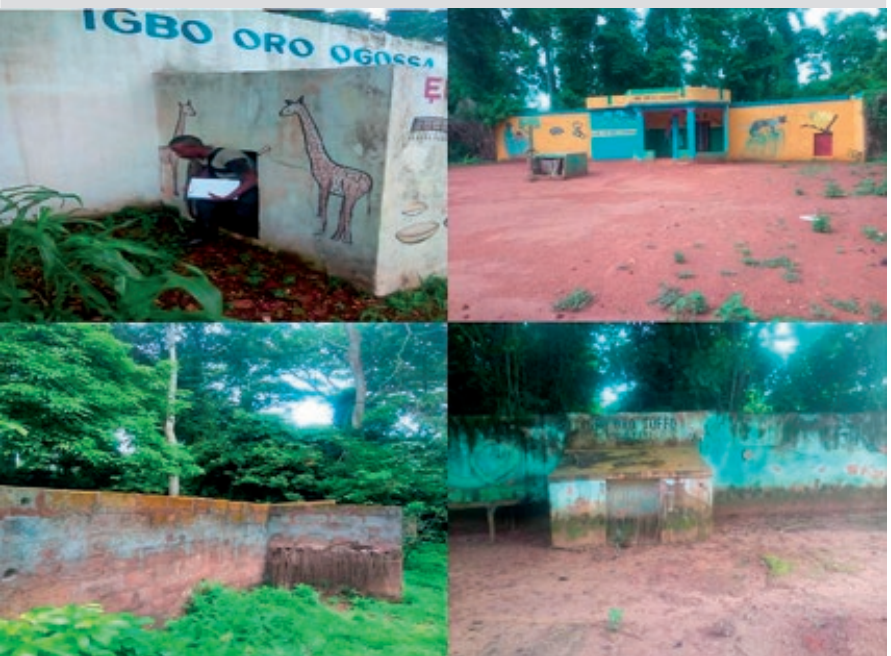

Photos 2.

Quelques couvents de forêts sacrées liées à la conservation de Triplochiton scleroxylon au Bénin.

Some sacred forest convents linked to the conservation of Triplochiton scleroxylon in Benin. Photos G. Ganka.

forêts sacrées abritant $T$. scleroxylon (photos 2) ainsi que les adeptes qui connaissent l'espèce et sont à même de citer différents critères de reconnaissance de celle-ci. Cette phase a montré que tous les adeptes des cultes associés à la conservation de $T$. scleroxylon connaissent et utilisent l'espèce. Ces adeptes sont tous de sexe masculin.

À la fin de la première phase, une permission a été demandée auprès du chef de village et du Adjana (chef dignitaire de Oro) de chaque site d'étude avant la conduite des enquêtes et ceci en respect du code d'éthique établi par la Société internationale d'ethnobiologie (International Society of Ethnobiology, 2006). Les enquêtés ont été identifiés et leur permission a été aussi obtenue avec l'aide des autorités locales avant l'administration du guide d'entretien conçu à cet effet.

Pour ce faire, les autorités locales et traditionnelles de chaque localité ont été informées de l'étude et des interprètes ont été engagés. Des photos des feuilles et des fruits de T. scleroxylon (photos 3) ont été montrées aux enquêtés pour faciliter la reconnaissance de l'espèce.

Compte tenu de la distribution de $T$. scleroxylon et de son caractère sacré au Bénin, l'approche d'échantillonnage non aléatoire par la technique de la boule de neige (Cochran, 1977) a été adoptée en vue d'identifier les informateurs clés parmi les acteurs des forêts sacrées du culte Oro et des divinités autres qu'Oro. Cette technique consiste à approcher un informateur compétent pour l'étude, qui, après avoir été enquêté, indique à son tour une autre personne compétente de la même communauté ; le processus évolue ainsi jusqu'à l'investigation de toutes les personnes compétentes pour le sujet d'étude. Cela nous a permis d'approcher directement des informateurs qui sont réputés détenir des connaissances sur T. scleroxylon au sein de leur communauté. En effet, l'estimation de l'effectif des

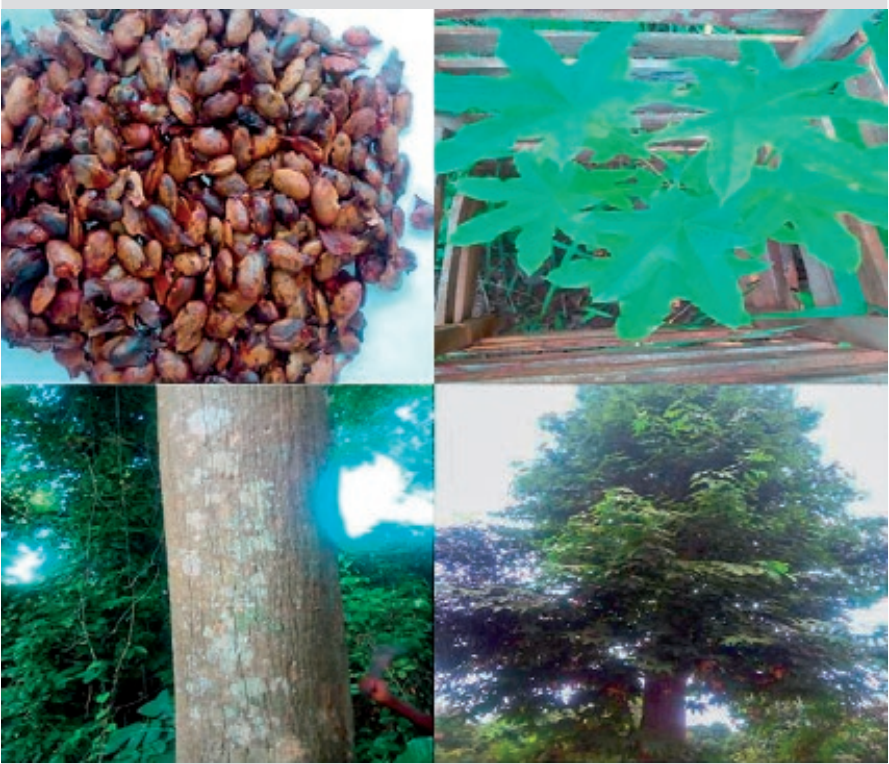

Photos 3.

Quelques organes (graine, feuille, tronc) et arbre

de Triplochiton scleroxylon.

Some organs (seed, leaf, trunk) and tree

of Triplochiton scleroxylon.

Photos G. Ganka.

informateurs clés a été considérée comme représentative lorsque la limite de saturation (point auquel plus aucun nom d'informateur clé n'est mentionné par le dernier enquêté) a été atteinte au terme de l'enquête (Cochran, 1977).

Le nombre d'enquêtés était de 297 au sein du groupe ethnolinguistique Nagot (72,26\%), 57 chez les Mahi $(13,87 \%), 3$ chez les Fon (0,73\%), 50 parmi les Kotafon (12,17\%) et 4 pour les Anii (0,97\%), soit 411 individus au total. Cependant, compte tenu de la faible représentativité des groupes ethniques Anii (0,97 \%) et Fon (0,73\%), les informateurs Anii et Fon n'ont pas été pris en compte dans les analyses statistiques. Ainsi, 404 enquêtés appartenant à trois groupes ethniques (Kotafon, Mahi, et Nagot) ont été retenus.

Les données ont été collectées à travers des entretiens semi-structurés individuels et des observations directes sur le terrain. Les données collectées sont structurées en trois parties. La première partie est relative aux caractéristiques de l'enquêté, notamment le groupe ethnique, l'âge et le groupe cultuel d'appartenance (communauté de culte à laquelle il appartient). La deuxième partie est relative aux appellations locales de l'espèce et leur signification ainsi qu'aux connaissances sur les usages spécifiques de l'espèce, et les parties ou organes utilisés. La troisième partie porte sur l'importance culturelle de la plante. Les données préliminaires ont permis d'identifier neuf catégories d'usage : alimentaire, médicinale, brosse végétale, culturelle, cultuelle, artisanale, bois énergie, bois d'œuvre et agropastoralisme. Pour évaluer l'importance des catégories d'usage et des organes, il a été demandé à chaque enquêté d'évaluer leur niveau d'importance. 
Pour les besoins de l'analyse, les enquêtés ont été répartis suivant l'âge (adultes, 18-60 ans ; personnes âgées, plus de 60 ans), l'appartenance à un culte lié à la conservation de $T$. scleroxylon et l'activité socioprofessionnelle (tableau I). Les catégories socioprofessionnelles identifiées étaient les agriculteurs (56,68\%), les sculpteurs (5,19\%) et les tradithérapeutes (les prêtres des divinités et guérisseurs traditionnels) $(24,26 \%)$. Une quatrième catégorie regroupe les autres activités $(13,63 \%)$ et inclut les fonctionnaires, mécaniciens, pépiniéristes, etc. (tableau I).

\section{Traitement et analyse des données}

Trois indices ethnobotaniques ont été utilisés pour l'analyse des données. Il s'agit de la fréquence relative de citation d'une utilisation $u\left(F R C_{u}\right)$, la valeur d'usage de la plante entière $\left(\mathrm{VU}_{\mathrm{T}}\right)$ et par organe $\left(\mathrm{VU}_{\text {org }}\right)$ et l'indice d'importance culturelle (IC). Ces indices ont permis d'examiner les connaissances des personnes interviewées sur l'espèce ainsi que son importance socioculturelle suivant les différents groupes.

La FRC mesure la popularité de chaque usage spécifique de la plante par les répondants d'un groupe donné (Friedman et al., 1986). Les valeurs élevées de la FRC pour une utilisation spécifique reflètent une popularité et généralement un consensus (partage des connaissances) pour cette utilisation de l'organe dans la communauté. Elle a été calculée par :
$F R C u=\frac{N u}{N} \times 100$

où $N_{u}$ représente le nombre de répondants qui ont déclaré l'utilisation $u$ et $N$ le nombre total de répondants.

Les utilisations rapportées par plus de $20 \%$ des répondants (FRC $>20 \%)$ ont été considérées comme des connaissances partagées (TRAMIL, 1989). Cependant, les connaissances rapportées par moins de $20 \%$ des répondants peuvent être importantes dans certains groupes ethnolinguistiques. Une utilisation $u$ a été considérée comme importante pour un groupe ethnolinguistique lorsque la $\mathrm{FRC}_{u}$ est supérieure à 5 \% (Gouwakinnou et al., 2011).

La $V_{\mathrm{T}}$ a été exprimée en termes de nombre moyen d'usages rapportés (Gomez-Beloz, 2002):

$V U_{T}=\frac{\sum_{i=1}^{N} U R_{i}}{N}$

où UR représente le nombre d'usages spécifiques rapporté par l'enquêté $i$ du groupe considéré et $N$ le nombre total d'enquêtés du groupe.

Compte tenu de la nature des données initiales de $\mathrm{VU}_{\mathrm{T}}$ (données de comptage), un modèle linéaire généralisé (GLM) basé sur la distribution binomiale négative a été utilisé pour tester l'influence des facteurs âge, groupe ethnolinguistique, cultuel et professionnel sur sa variation. Le modèle saturé a été d'abord spécifié, puis progressivement simplifié en utilisant la valeur du critère d'information d'Akaike (Akaike, 1973 ; Johnson et Omland, 2004) jusqu'à la

Tableau I.

Répartition des enquêtés par groupes ethnolinguistiques et cultuels.

Distribution of respondents by ethnolinguistic and cultural groups.

\begin{tabular}{|c|c|c|c|c|}
\hline $\begin{array}{l}\text { Groupe ethnolinguistique } \\
\text { et domaine d'activité }\end{array}$ & Groupe sociocultuel & Adulte & Vieux & $\begin{array}{l}\text { Proportion } \\
\text { (\%) }\end{array}$ \\
\hline \multirow[t]{2}{*}{$\begin{array}{l}\text { Groupe ethnolinguistique } \\
\text { Nagot }\end{array}$} & Acteur du culte Oro & 92 & 33 & 72,26 \\
\hline & Acteur d'autres divinités & 97 & 75 & \\
\hline \multirow[t]{2}{*}{ Mahi } & Acteur du culte Oro & 20 & 6 & 13,87 \\
\hline & Acteur d'autres divinités & 21 & 10 & \\
\hline \multirow[t]{2}{*}{ Kotafon } & Acteur du culte Oro & 0 & 0 & 12,17 \\
\hline & Acteur d'autres divinités & 33 & 17 & \\
\hline \multirow[t]{2}{*}{$\begin{array}{l}\text { Domaine d'activité } \\
\text { Agriculture }\end{array}$} & Acteur du culte Oro & 42 & 19 & 56,7 \\
\hline & Autre acteur & 106 & 62 & \\
\hline \multirow[t]{2}{*}{ Tradithérapie } & Acteur du culte Oro & 52 & 22 & 24,2 \\
\hline & Autre acteur & 4 & 20 & \\
\hline \multirow[t]{2}{*}{ Autre } & Acteur du culte Oro & 12 & 0 & 13,6 \\
\hline & Autre acteur & 26 & 18 & \\
\hline \multirow[t]{2}{*}{ Sculpture } & Acteur du culte Oro & 4 & 0 & 5,2 \\
\hline & Autre acteur & 17 & 0 & \\
\hline
\end{tabular}


Tableau II.

Appellations locales de Triplochiton scleroxylon au Bénin. Local names for Triplochiton scleroxylon in Benin.

\begin{tabular}{l|l|l|}
$\begin{array}{l}\text { Groupe } \\
\text { ethnolinguistique }\end{array}$ & $\begin{array}{l}\text { Appellation } \\
\text { utilisée }\end{array}$ & Signification \\
\hline Kotafon & Athiwé & Bois blanc \\
\hline Mahi & Whètin & \\
\hline Nagot/Yoruba & Aréré ; Egui Aréré & L'arbre de l'étage dominant de toute forêt \\
\hline Egui alassa & \\
\hline Egui Oro & L'arbre sacré du fétiche Oro \\
\hline Egui ogù & L'arbre sacré \\
\hline
\end{tabular}

\section{Résultats}

\section{Appellations locales}

Les appellations utilisées par les enquêtés pour désigner T. scleroxylon et leur signification varient suivant les groupes ethnolinguistiques rencontrés (tableau II).

\section{Diversité des usages de Triplochiton scleroxylon}

Triplochiton scleroxylon présente 36 usages spécifiques catégorisés en neuf types d'utili-

sélection du meilleur modèle (modèle ayant le plus faible nombre de facteurs avec des effets significatifs) (Johnson et Omland, 2004).

La $V U_{\text {org }}$ est exprimée en termes de nombre moyen d'usages par enquêté et par organe. Elle a été calculée en utilisant la formule ci-dessous (Gomez-Beloz, 2002) :

$V U_{\text {org }}=\frac{\sum_{i=1}^{N} U R_{\text {org }, i}}{N}$

où VU $U_{\text {org }}$ est la valeur d'usage d'un organe donné, $U R_{\text {org, } i}$ est le nombre d'usages mentionné pour cet organe par l'enquêté $i$ et $N$ est le nombre de personnes enquêtées du groupe.

Les organes ayant une $V U_{\text {org }}$ élevée sont considérés comme les parties ayant le plus d'usages connus et implicitement les organes les plus exploités par la population. La $V U_{\text {org }}$ a été comparée entre organes en utilisant un modèle linéaire général (GLM) basé sur la distribution de Poisson.

L'indice d'importance culturelle (IC) désigne la valeur allouée culturellement à la plante ainsi que celle rattachée à ses organes (Houehanou et al., 2011). L'IC des catégories d'usage $\left(I C_{c u}\right)$ et des organes $\left(I C_{\text {org }}\right.$ ) a été calculée en utilisant les deux formules ci-dessous (Houehanou et al., 2011) :

$I C_{\text {cug }}=\frac{\sum_{i=1}^{N} S_{c u, i}}{N}$

$I C_{\text {org }}=\frac{\sum \sum \sum_{i=1}^{N} S_{\text {org, } i}}{N}$

Pour une catégorie d'usage donnée, $S_{c u, i}$ est le score d'importance culturelle attribué par l'enquêté $i$ à cette catégorie. Pour un organe donné, $S_{\text {org, }}$ est le score d'importance culturelle attribué par l'enquêté $i$ à cet organe. $N$ est le nombre de personnes enquêtées du groupe. Ces scores ont été obtenus par la conversion des niveaux d'importance en termes de " pas important », « peu important », « moyennement important » et « très important », respectivement en $0,1,2$ et 3 . Des diagrammes en radar ont été construits pour analyser la variation de l'IC $C_{c u}$ et de l'I $C_{\text {org }}$ suivant les groupes ethniques. Toutes les analyses statistiques ont été réalisées sous le logiciel R version 3.5.0 (R Core Development, 20201).

1 https://cran.r-project.org/bin/windows/base/old/3.5.0/ sation (tableau III). Seul l'usage comme bois énergie présente une faible fréquence de citation (FRC $=0,97 \%$ ). L'usage culturel est le plus rapporté par les enquêtés (FRC =96,1\%). La diversité des usages de T. scleroxylon varie en fonction des organes de la plante. Les organes les plus impliqués dans les usages sont les feuilles (11 usages), les écorces (6), les racines (6), le tronc (8) et les tiges et branches (5) (tableau III).

\section{Usages médicinaux}

Triplochiton scleroxylon est utilisé dans le traitement de 14 maladies et symptômes. Le recours à $T$. scleroxylon dans le traitement des troubles digestifs et des affections gynécologiques est le plus connu de la population, respectivement $53,3 \%$ et $38,9 \%$ des enquêtés (tableau IV). T. scleroxylon est aussi sollicité pour soigner de multiples maladies : diabète (17,3\%), épilepsie (10,2 \%), varicelle (12,9\%), asthme (23,3\%), prostate (17,8 \%) et cancer du sein (10,7 \%), mais il reste peu utilisé pour le traitement des maladies cardiaques (3,6\%).

\section{Relation entre les facteurs ethnolinguistiques, cultuels et socioprofessionnels et la valeur d'usage de Triplochiton scleroxylon}

Les connaissances et usages de T. scleroxylon varient significativement suivant les groupes ethnolinguistiques (GSC) et la catégorie d'adeptes (CA). Le facteur âge n'a pas d'effet significatif sur la variation des connaissances et usages de T. scleroxylon.

La valeur d'usage totale de T. scleroxylon était plus élevée chez les Nagot $(12,14 \pm 0,34)$ suivis des Mahi $(7,71 \pm 0,56)$ (figure 1a). Au sein des groupes cultuels, les acteurs du culte Oro utilisent plus l'espèce que les acteurs des autres divinités (figure 2b). Les tradithérapeutes et les sculpteurs détiennent également plus de connaissances que ceux des autres domaines d'activité (figure 2c).

Afin d'évaluer la relation entre les facteurs sociodémographiques et le niveau de connaissance des usages de T. scleroxylon, différents modèles ont été testés (tableau V). 


\section{Tableau III.}

Catégorie d'usage de Triplochiton scleroxylon au Bénin.

Category of use of Triplochiton scleroxylon in Benin.

\begin{tabular}{|c|c|c|c|}
\hline $\begin{array}{l}\text { Catégorie } \\
\text { d'usage }\end{array}$ & $\begin{array}{l}\text { FRC } \\
\text { (\%) }\end{array}$ & Observations & Parties utilisées \\
\hline Alimentaire & 71 & Utilisé pour préparer la sauce gluante & Feuille \\
\hline Médicinale & 72,7 & Traitement des maladies et symptômes & Feuille, racine, écorce \\
\hline Brosse végétale & 18 & Utilisé comme cure-dent & Tige/branche, racine \\
\hline Culturelle & 96,1 & $\begin{array}{l}\text { Émondage des feuilles par le fétiche Oro, usage du bois dans la fabrication des } \\
\text { tam-tams et masque du culte « Guèlèdè » et de bâton de guide de vodoun Egoun-goun }\end{array}$ & $\begin{array}{l}\text { Feuille, fruit/graine, racine, } \\
\text { branche, tronc }\end{array}$ \\
\hline Cultuelle & 36,5 & $\begin{array}{l}\text { Usage dans les cérémonies de sortie de fétiche Oro, usage dans l'art divinatoire, } \\
\text { la fabrication de tam-tam sacré Oro, fabrication des statuettes de jumeaux et de protection } \\
\text { spirituelle et autres usages spirituels dans les couvents du culte Oro }\end{array}$ & Feuille, tronc, branche/tige, racine \\
\hline Artisanale & 74,4 & Fabrication d'objets d'art, de palettes de cuisine & Branche, tronc \\
\hline Bois énergie & 0,9 & Utilisé comme bois de feu et charbon & Branche, tronc \\
\hline Bois d'œuvre & 88,1 & Fabrication de meubles, pirogues monoxyles, objets sculptés, mortiers, tam-tams & Tronc \\
\hline Agropastoralisme & 5,1 & Alimentation et soins du bétail & Feuille \\
\hline
\end{tabular}

\section{Tableau IV.}

Usages médicinaux de Triplochiton scleroxylon au Bénin.

Medicinal uses of Triplochiton scleroxylon in Benin.

\begin{tabular}{|c|c|c|c|c|c|c|}
\hline $\begin{array}{l}\text { Catégorie } \\
\text { d'affection }\end{array}$ & $\begin{array}{l}\text { Maladie et } \\
\text { symptôme }\end{array}$ & Organes & $\begin{array}{l}\text { Mode de } \\
\text { préparation }\end{array}$ & $\begin{array}{l}\text { Mode } \\
\text { d'admission }\end{array}$ & $\begin{array}{l}\text { Groupe } \\
\text { sociolinguistique }\end{array}$ & FRC \\
\hline \multirow[t]{2}{*}{ Digestive } & Constipation & Feuille & Décoction & Boisson & Anii ; Fon ; Kotafon ; Mahi ; Nagot & 32,6 \\
\hline & Maux de ventre & Feuille & Décoction & Boisson & Anii ; Fon ; Kotafon ; Mahi ; Nagot & 53,3 \\
\hline \multirow[t]{5}{*}{ Gynécologique } & Accouchement compliqué & Feuille & Décoction & Boisson & Anii ; Fon ; Kotafon ; Mahi ; Nagot & 38,9 \\
\hline & Irrégularité des menstrues & Feuille & Trituration & Boisson & & 31,4 \\
\hline & Menstrues intermittentes & Feuille & Décoction & Boisson & & 30,6 \\
\hline & Pertes blanches & Feuille & Trituration & Boisson & & 28,7 \\
\hline & Fibrome & Racine & Poudre & Mélange alcoolique & Nagot; Mahi & 18,2 \\
\hline Cardiologique & Hypotension artérielle & Écorce & Décoction & Boisson & Nagot & 3,6 \\
\hline Endocrinienne & Diabète & Écorce & Décoction & Boisson & Nagot; Mahi & 17,3 \\
\hline Neurologique & Épilepsie & Racine & Poudre & Lapement & Nagot & 10,2 \\
\hline Dermatologique & Varicelle & Écorce & Poudre & Application locale & Nagot;Mahi & 12,9 \\
\hline Cancéreuse & Cancer du sein & Écorce & Poudre & Mélange alimentaire & Nagot & 10,7 \\
\hline \multirow[t]{2}{*}{ Autres } & Asthme & Écorce & Poudre & Lapement & Nagot; Mahi & 23,3 \\
\hline & Prostate & Écorce & Macération & Boisson & Nagot; Mahi & 17,8 \\
\hline
\end{tabular}

Le modèle incluant l'interaction des facteurs groupe ethnolinguistique (GSC) et catégorie d'adeptes (CA) est le modèle adéquat du fait de sa faible valeur de l'AIC (tableau V). La variation du degré de connaissance des usages de T. scleroxylon est seulement déterminée par les groupes cultuels et ethnolinguistiques.
Les connaissances des usages de $T$. scleroxylon sont significativement plus élevées chez les Nagot et les Mahi que chez les Kotafon. Les adeptes du culte Oro possèdent plus de connaissances sur les usages de T. scleroxylon que les adeptes des autres divinités (tableau VI). 

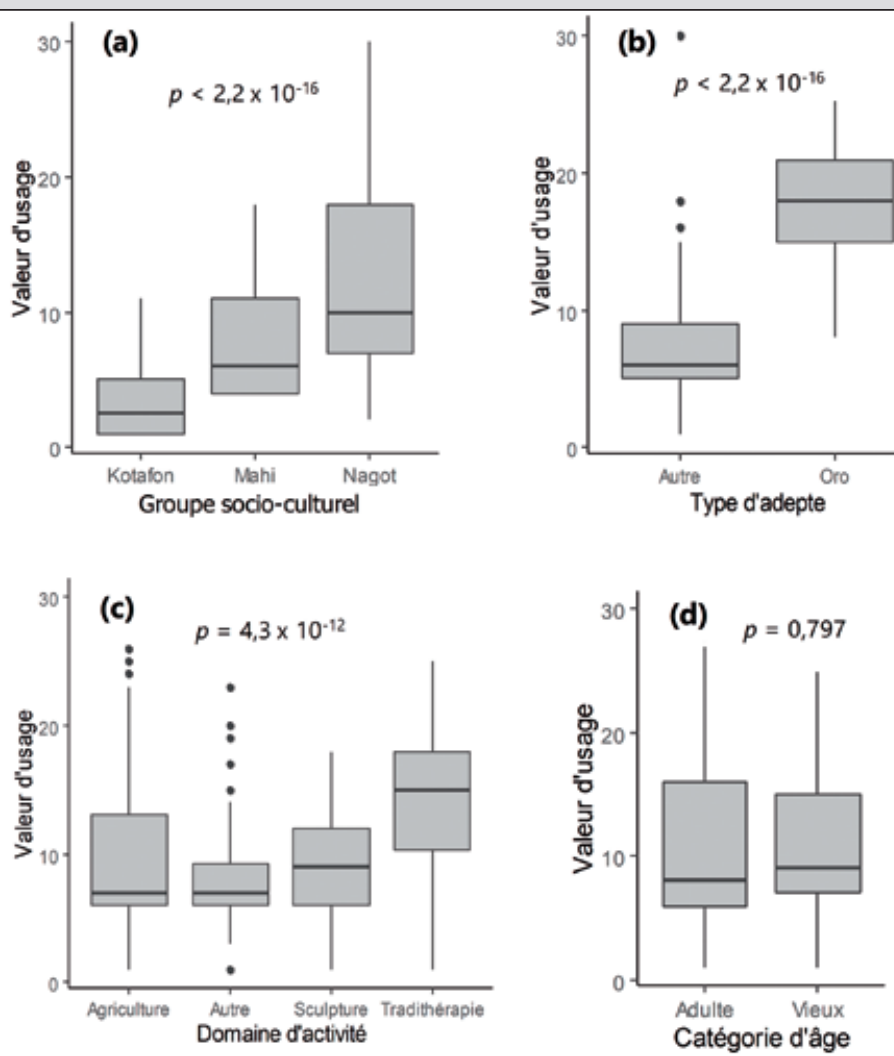

Figure 2.

Variation des valeurs d'usages ethnobotaniques de Triplochiton scleroxylon suivant les groupes sociodémographiques. Variation in ethnobotanical use values of Triplochiton scleroxylon according to socio-demographic groups.

Tableau V.

Sélection du modèle parcimonieux.

Selection of the parsimonious model.

\begin{tabular}{|c|c|c|}
\hline Modèles candidats & AIC & $\triangle \mathrm{AIC}$ \\
\hline $\mathrm{GSC}+\mathrm{CA}+\mathrm{GSC}: \mathrm{CA}$ & 2123,6 & 0 \\
\hline$C A+\hat{A} g e+C A:$ Âge & 2226,8 & 103,2 \\
\hline CA + DomAct + CA : DomAct & 2228 & 104,4 \\
\hline $\mathrm{CA}$ & 2238,1 & 114,5 \\
\hline GSC + DomAct + GSC : DomAct & 2351 & 227,4 \\
\hline GSC + Âge + GSC : Âge & 2407,8 & 284,2 \\
\hline GSC & 2415 & 291,4 \\
\hline DomAct + Âge + DomAct : Âge & 2520,1 & 396,5 \\
\hline DomAct & 2522,5 & 398,9 \\
\hline Âge & 2570,5 & 446,9 \\
\hline
\end{tabular}

\section{Variation des VUR de Triplochiton scleroxylon au sein de la communauté Nagot}

Les deux catégories de cultes existent au sein du groupe ethnolinguistique Nagot, contrairement aux groupes Kotafon et Mahi caractérisés uniquement soit par le culte Oro (pour les Mahi), soit pour une autre divinité (chez les Kotafon). Au sein du groupe ethnolinguistique Nagot, les adeptes du culte Oro ont plus de connaissances sur l'usage de $T$. scleroxylon que ceux des autres divinités $(p<0,001)$ avec un nombre d'usages moyen de 17,90 $\pm 3,7$ et un nombre d'usages médian de 18 contre $7,9 \pm 3,0$ usages moyens pour 7 usages médians.

\section{Valeur d'usage des organes de Triplochiton scleroxylon}

Plusieurs parties de T. scleroxylon sont sollicitées quotidiennement pour divers usages : feuilles, fleurs, fruits/grains, racines, écorce, tige et bois. Les valeurs d'usage varient entre les organes de l'espèce. La feuille est l'organe le plus utilisé, suivie du bois, de la tige et des racines (figure 3 ).

La figure 4 présente les valeurs d'usage moyennes des différentes parties de T. scleroxylon suivant les groupes ethnolinguistiques (figure 4). Les groupes qui utilisent le plus les organes de T. scleroxylon sont les Nagot et les Mahi (figure 4). Cependant, Il faut noter que l'usage de toutes les parties de l'espèce est beaucoup plus prononcé parmi les Nagot que chez les autres groupes ethnolinguistiques (figure 4).

\section{Importance culturelle de Triplochiton scleroxylon}

La figure 5 présente la variation de l'importance culturelle de T. scleroxylon suivant les groupes ethnolinguistiques au Bénin. Les Nagot accordent une importance relativement plus marquée à l'ensemble des usages de l'espèce que les autres groupes ethnolinguistiques (figure 5). Cependant, il faut noter que les usages culturels, cultuels et ceux comme le bois d'œuvre de T. scleroxylon sont beaucoup plus marqués pour cette communauté. La plupart des groupes ethnolinguistiques accordent davantage d'importance à l'usage médicinal qu'à celui du bois d'œuvre de l'espèce. Les usages de l'espèce comme le bois énergie et l'agropastoralisme sont quasiment sans importance pour les enquêtés (figure 5).

L'importance culturelle des organes de T. scleroxylon diffère suivant les groupes ethnolinguistiques (figure 5). La plupart des parties de l'espèce ont de l'importance pour les groupes ethnolinguistiques Nagot et Mahi. Les feuilles, les racines et le bois de $T$. scleroxylon sont culturellement plus importants que toutes les autres parties de la plante pour cette communauté. Le bois de l'espèce a de l'importance pour tous les groupes ethnolinguistiques rencontrés. 


\section{Discussion}

Tableau VI.

Résultats de la régression binomiale négative sur les valeurs d'usage. Results of the negative binomial regression on use values.

\begin{tabular}{l|c|c|c|c|}
\hline & Estimation & $\begin{array}{c}\text { Erreur } \\
\text { type }\end{array}$ & $\begin{array}{c}\text { Valeur } \\
\text { de Z }\end{array}$ & $\begin{array}{c}\text { Probabilité } \\
(>|\mathbf{z}| \mathbf{)}\end{array}$ \\
\hline Intercept & 1,23547 & 0,07795 & 15,85 & $<0,001^{\star * *}$ \\
\hline Mahi & 0,80825 & 0,09262 & 8,727 & $<0,001^{\star * *}$ \\
\hline Nagot & 0,83814 & 0,08296 & 10,103 & $<0,001^{\star * *}$ \\
\hline Adepte du culte Oro & 0,81141 & 0,03686 & 22,015 & $<0,001^{\star * *}$ \\
\hline
\end{tabular}

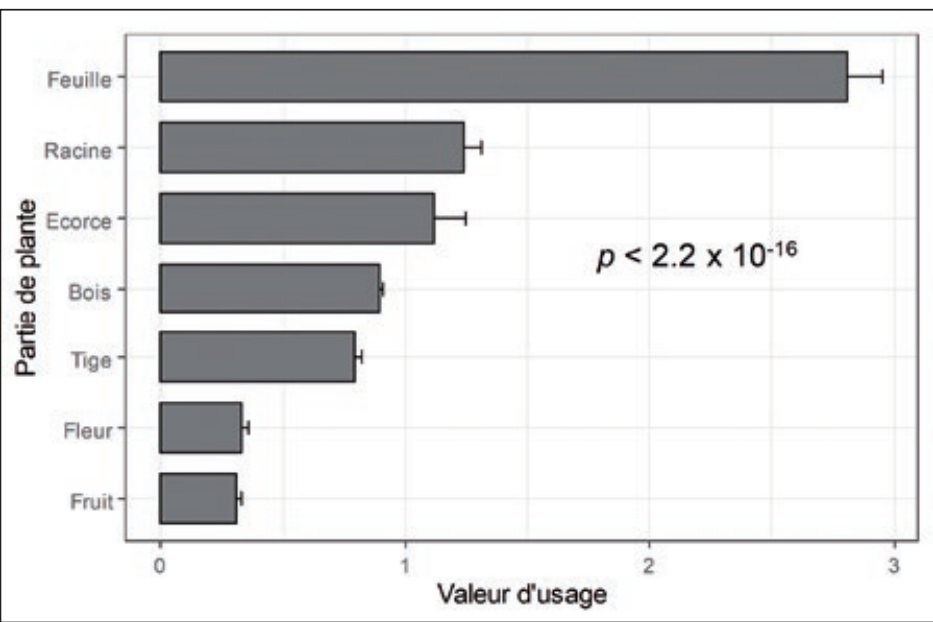

Figure 3.

Importance d'usage des parties de plante de Triplochiton scleroxylon.

Importance of using the plant parts of Triplochiton scleroxylon.

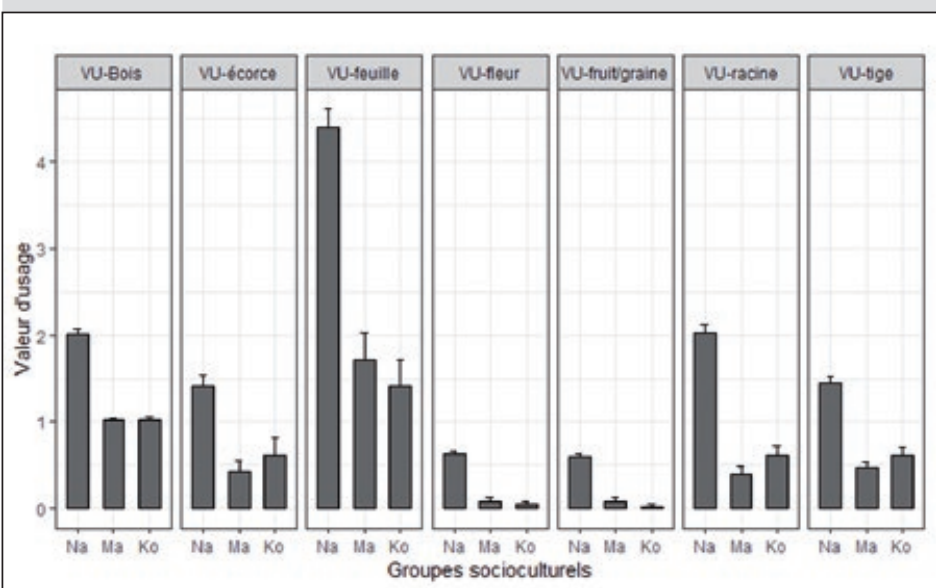

Figure 4.

Valeur d'usage des organes ou parties de Triplochiton scleroxylon suivant les groupes ethnolinguistiques. Use value of organs or parts of Triplochiton scleroxylon according to ethnolinguistic groups.

$\mathrm{Na}=$ Nagot $; \mathrm{Ma}=$ Mahi $; \mathrm{Ko}=$ Kotafon
Les perceptions sur l'appellation de l'espèce varient suivant les groupes ethnolinguistiques rencontrés. Par exemple, les Nagot désignent $T$. scleroxylon sous le nom de " Aréré » comme étant l'arbre de l'étage dominant de la forêt, « Egui Oro » l'arbre qui symbolise le fétiche Oro et « Egui ogù » pour son statut d'arbre sacré. Le nom vernaculaire d'une espèce est donc la révélation d'une tradition donnée (Assogba et al., 2017) ou du trait morphologique de l'espèce (Achigan-Dako et al., 2010 ; Fandohan et al., 2017 ; Assogba et al., 2017). L'appellation « Egui Oro » ou « Egui ogù » en milieu Nagot suggère qu'il s'agit d'une espèce ayant un fort degré de signification cultuelle au sein de ce groupe ethnolinguistique.

Les usages les plus connus par les enquêtés, qui sont les traitements des affections gynécologiques (accouchement difficile, irrégularité des menstrues et menstrues intermittentes, pertes blanches, fibrome ou kyste et myomes) et de l'épilepsie, étaient absents de la littérature. Ces usages pourraient offrir des perspectives de recherche intéressantes en phytochimie.

Le résultat montrant que les acteurs du culte Oro ont plus de connaissances sur T. scleroxylon que les acteurs d'autres divinités pourrait être lié au contact régulier avec l'espèce à travers les cérémonies rituelles du culte Oro, et cela confirme l'hypothèse selon laquelle les savoirs et le degré de connaissance sur les plantes dépendent des cultures et des us et coutumes des peuples (Sundriyal et al., 2004). Cette hypothèse est d'autant plus vérifiée que, en plus d'être du même groupe ethnolinguistique, les adeptes du culte Oro ont plus de connaissances sur l'usage de T. scleroxylon que les Nagot, acteurs d'autres divinités des écosystèmes sacrés abritant l'espèce.

Les connaissances traditionnelles sur T. scleroxylon n'ont pas varié suivant les catégories d'âge (figure 2). Bien que les vieux aient plus recours à l'espèce que les adultes, la variation des connaissances n'est pas significative. L'hypothèse de Souto et Ticktin (2012), qui prédit que la connaissance traditionnelle dépend de l'âge (Gaoué et al., 2017), est ainsi mise en défaut. Les résultats obtenus pourraient se justifier par la position qu'occupent les adultes au sein de la tradition du culte Oro, qui influence significativement la connaissance et l'usage de T. scleroxylon. En effet, le trône de Adjana (responsable de la tradition Oro) est spécialement destiné à certaines familles. Ainsi, à partir de 18 ans, tout membre de la famille peut être choisi par les dieux après consultation pour occuper le poste de Adjana. Les secrets et les connaissances liés à ce rang devront lui être transmis par ses pairs. Il découle alors des résultats obtenus une hypothèse selon laquelle le degré des connaissances endogènes sur les espèces sacrées est fonction du niveau de responsabilité de l'informateur dans la tradition considérée. Elle se justifie d'autant plus que, en considérant les trois catégories d'âge, la valeur d'usage moyenne 

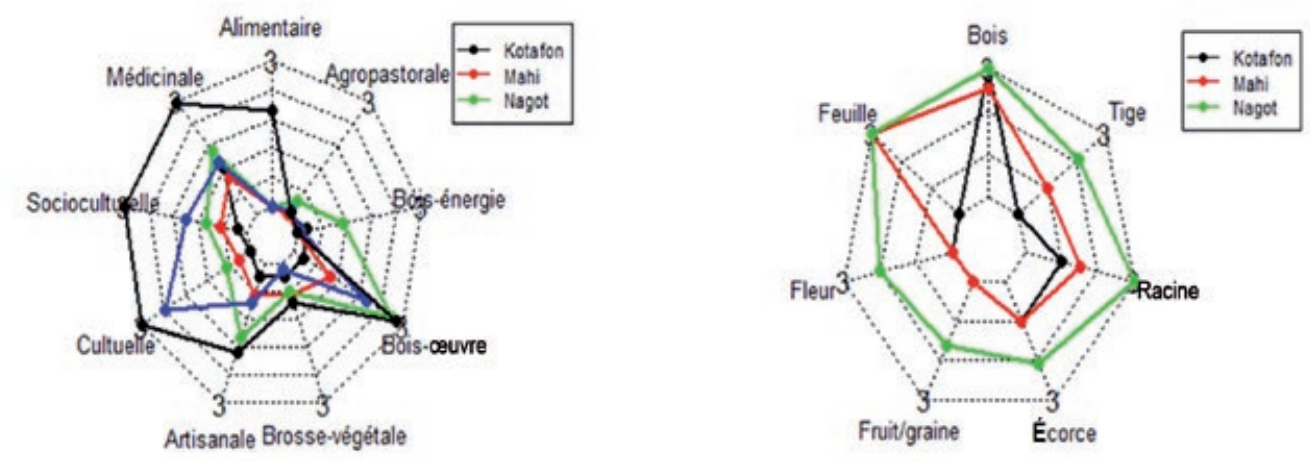

Figure 5.

Importance culturelle des catégories d'usage et des organes

de Triplochiton scleroxylon en fonction des groupes ethnolinguistiques.

Cultural significance of use categories and organs of Triplochiton

scleroxylon according to ethnolinguistic groups.

des sept enquêtés ayant moins de 30 ans (jeunes) est supérieure à celle des adultes (âge supérieur à 30 ans) et à celle des vieux. Cette hypothèse pourrait cependant être approfondie par des travaux complémentaires en ethnobiologie sur d'autres espèces rituelles.

La singularité des connaissances et usages de T. scleroxylon par les acteurs du culte Oro serait en lien avec l'unicité des places qu'occupe chaque organe de la plante dans l'identité et la stabilité du culte Oro. Oro est un culte qui caractérise potentiellement l'identité du grand groupe des Yoruboïdes du Bénin et du Nigeria. Les Yoruboïdes regroupent les Yoruba, Nagot, Idatcha et apparentés. Il est donc important d'explorer les impacts du déclin de T. scleroxylon sur l'identité et la stabilité culturelle des peuples qui en dépendent, en relation avec les implications écologiques pour la conservation des écosystèmes sacrés. L'identification et l'appréciation du réseau complexe de connexions entre la diversité biologique et culturelle/cultuelle, l'importance de la résilience et la réponse adaptative dans les communautés socio-écologiques sont très prisées dans les efforts de conservation et de restauration (Garibaldi et Turner, 2004). La multiplicité des usages de l'espèce pourrait avoir aussi des impacts sur son écologie puisqu'il est montré que les effets de l'anthropisation peuvent impacter la structure et la stabilité des populations de plantes préférées (Gaoué et Ticktin, 2007). La conséquence écologique la plus immédiate de la pression humaine sur les essences prisées est l'altération du taux de survie, de la croissance et de la reproduction de ces individus. Cependant, l'existence des tabous relatifs à l'espèce, liés à son statut d'arbre sacré et vénéré, pourrait aussi contribuer à sa conservation. En effet, selon l'hypothèse des tabous comme stratégie de conservation, les espèces sont rendues taboues pour les protéger de la surexploitation et de l'extinction (Colding et Folke,1997). Les cultes étant les seuls producteurs de tabous, leur implication dans la préservation des ressources naturelles constitue une perspective à ne pas négliger dans les stratégies de conservation de la biodiversité. Compte tenu de l'importance de l'espèce et de son usage dans les cérémonies et rituels, des études sur l'impact de la prévalence de ces cérémonies et des tabous sur la structure démographique et spatiale des peuplements de $T$. scleroxylon pourraient améliorer l'état des connaissances sur l'espèce.

\section{Conclusion}

Cette étude a porté sur l'importance des cultes dans la préservation des espèces d'arbre et a été conduite auprès des sages, dignitaires et autres informateurs clés des écosystèmes sacrés abritant Triplochiton scleroxylon au Bénin. Elle a permis de construire une base de données sur les connaissances et les usages traditionnels de l'espèce dans ce pays. Les résultats obtenus témoignent de la nécessité d'investir dans la sylviculture des espèces forestières qui occupent une place de choix dans l'ethnosphère africaine pour le renforcement de système socio-écologique. En effet, l'usage de T. scleroxylon est connu de plusieurs groupes ethnolinguistiques, son importance variant suivant ces différents groupes, avec une particularité liée aux acteurs du culte Oro qui détiennent plus de connaissances sur l'usage de l'espèce et de ses différentes composantes. Une étude de l'impact de la disparition de T. scleroxylon sur la diversité bioculturelle est donc nécessaire. Compte tenu de la place de T. scleroxylon dans la tradition, il est aussi important d'évaluer l'impact de la prévalence des cérémonies rituelles et des tabous liés à l'espèce sur la structure démographique et spatiale de T. scleroxylon. Les usages de l'espèce en médecine traditionnelle pour le traitement des affections gynécologiques et de l'asthme suggèrent aussi des études de « screening " phytochimique des feuilles, des racines et des écorces de $T$. scleroxylon pour plus d'informations sur les propriétés pharmacologiques de l'espèce. 


\section{Références}

Achigan-Dako E. G., Pasquini M. W., Assogba-Komlan F., N'Danikou S., Yédomonhan H., Dansi A., et al., 2010. Traditional vegetables in Benin. Cotonou, Institut national des recherches agricoles du Bénin, CENAP, $282 \mathrm{p}$.

Adomou A. C., 2005. Vegetation patterns and environmental gradients in Benin. Implications for biogeography and conservation. PhD thesis, Wageningen University, Wageningen, The Netherlands, 136 p. https://library.wur.nl/WebQuery/wurpubs/ fulltext/121707

Adomou C. A., Agbani O. P., Sinsin B., 2011. Plants. In: Neuenschwander P., Sinsin B., Goergen G. (eds). Nature Conservation in West Africa: Red List for Benin. Ibadan, Nigeria, International Institute of Tropical Agriculture, 21-46.

Akaike H., 1973. Information theory as an extension of the maximum likelihood principle. In: Petrov B. N., Csaki F. (eds). Second International Symposium on Information Theory. Budapest, Hungary, Akademiai Kiado, 267-281.

Akoegninou A., Van der Burg W. J., Van der Maesen L. J. G., Adjakidjè V., Essou J. P., Sinsin B., et al. (éds), 2006. Flore analytique du Bénin. Leiden, Pays-Bas, Backhuys Publishers, 1034 p. https:// library.wur.nl/WebQuery/wurpubs/fulltext/281595

Assogba G. A., Fandohan A. B., Salako V. K., Assogbadjo A. E., 2017. Usages de Bombax costatum (Malvaceae) dans les terroirs riverains de la Réserve de biosphère de la Pendjari, République du Bénin. Bois et Forêts des Tropiques, 333 (3) : 17-29. https://doi. org/10.19182/bft2017.333.a31465

Bayol N., Demarquez B., De Wasseige C., Ebaa A. R., Fisher J. F., Nasi R., et al., 2012. Forest management and the timber sector in Central Africa. Luxembourg, Publications Office of the European Union, 19 p. https://www.researchgate.net/profile/Robert Nasi/ publication/232660071_Forest_Management_and_the_Timber sector_in_Central_Africa/links/0046352c201965d00a000000.pdf

Cavendish W., 2000. Empirical regularities in the poverty-environment relationship of rural household: evidence from Zimbabwe. World Development, 28 (11): 1979-2003. https://doi. org/10.1016/S0305-750X(00)00066-8

Cochran W. G., 1977. Sampling techniques. 3rd edition. New York, USA, Wiley, $448 \mathrm{p}$.

Colding J., Folke C., 1997. The relations among threatened species, their protection, and taboos. Ecology and Society, 1 (1): 6. https://www.ecologyandsociety.org/vol1/iss1/art6/

de Albuquerque U. P., Soldati G. T., Sieber S. S., Ramos M. A., De Sá J. C., De Souza L. C., 2011. The use of plants in the medical system of the Fulni-ô people (NE Brazil): A perspective on age and gender. Journal of Ethnopharmacology, 133: 866-873. https:// doi.org/10.1016/i.jep.2010.11.021

Fandohan A. B., Chadare F. J., Gouwakinnou G. N., Tovissode C. F., Bonou A., Djonlonkou S. F. B., et al., 2017. Usages traditionnels et valeur économique de Syncepalum dulcificum au Sud-Bénin. Bois et Forêts des Tropiques, 332 (2) : 18-30. https://doi. org/10.19182/bft2017.332.a31330

FAO, 2018. La situation des forêts du monde 2018. Les forêts au service du développement durable. Rome, Italie, FAO, 158 p. https://www.fao.org/documents/card/fr/c/19535FR/
Fayolle A., Engelbrecht B., Freycon V., Mortier F., Swaine M., Réjou-Méchain M., et al., 2012. Geological substrates shape tree species and trait distributions in African moist forests. PLoS ONE 7, e42381. https://doi.org/10.1371/journal.pone.0042381

Friedman J., Yaniv Z., Dafni A., Palewith D., 1986. A preliminary classification of the healing potential of medicinal plants, based on a rational analysis of an ethnopharmacological field survey among Bedouins in the Negev Desert, Israel. Journal of Ethnopharmacology, 16 (2-3): 275-287. https://doi. org/10.1016/0378-8741(86)90094-2

Gaoué O. G., Ticktin T., 2007. Patterns of harvesting foliage and bark from the multipurpose tree Khaya senegalensis in Benin: variation across ecological regions and its impacts on population structure. Biological Conservation, 137 (3): 424-436. https:// doi.org/10.1016/J.BIOCON.2007.02.020

Gaoué O. G., Coe M. A., Bond M., Hart G., Seyler B. C., McMillen H., 2017. Theories and major hypotheses in ethnobotany. Economic Botany, 71 (3): 269-287. https://www.fs.fed.us/nrs/pubs/ $\mathrm{jrnl} / 2017 / \mathrm{nrs} 2017$ gaoue_001.pdf

Garibaldi A., Turner N., 2004. Cultural keystone species: Implications for ecological conservation and restoration. Ecology and Society, 9 (3): 1. https://www.ecologyandsociety.org/vol9/iss3/art1 Gomez-Beloz A., 2002. Plant use knowledge of the Winikina Warao: The case for questionnaires in ethnobotany. Economic Botany, 56 (3): 231-241. https://www.jstor.org/stable/4256576

Gouwakinnou G. N., Lykke A. M., Assogbadjo A. E., Sinsin B., 2011. Local knowledge, pattern and diversity of use of Sclerocarya birrea. Journal of Ethnobiology and Ethnomedicine, 7: 8. https:// doi.org/10.1186/1746-4269-7-8

Hall J. B., Bada S. O., 1979. The distribution and ecology of Obeche (Triplochiton Scleroxylon). Journal of Ecology, 67: 543564. https://www.jstor.org/stable/2259111

Hanazaki N., Herbst D. F., Marques M. S., Vandebroek I., 2013. Evidence of the shifting baseline syndrome in ethnobotanical research. Journal of Ethnobiology and Ethnomedicine, 9: 75. https://doi.org/10.1186/1746-4269-9-75

Houehanou T. D., Assogbadjo A. E., Glèlè Kakaï R., Houinato M., Sinsin B., 2011. Valuation of local preferred uses and traditional ecological knowledge in relation to tree multipurpose tree species in Benin (West Africa). Forest Policy and Economics, 13 (7): 554-562. https://bec.uac.bj/uploads/publication/3335705f39c976b9c5c7e9f2e91a1d49.pdf

International Society of Ethnobiology, 2006. ISE Code of Ethics (With 2008 additions). ISE, 12 p. https://www.ethnobiology.net/what-we-do/core-programs/ise-ethics-program/ code-of-ethics/

Johns T., Mohoro E. B., Sanaya P., 1996. Food plants and masticants of the Batemi of Ngorongoro District, Tanzania. Economic Botany. 50: 115-121.

Johnson J. B., Omland K. S., 2004. Model selection in ecology and evolution. Trends in Ecology and Evolution, 19 (2): 101108. http://faculty.washington.edu/skalski/classes/QERM597/ papers/Johnson\%20and\%200mland.pdf 
Kalinganire A., Weber J. C., Uwamariya A., Kone B., 2008. Improving rural livelihoods through domestication of indigenous fruit trees in the parklands of the Sahel. In: Akinnifesi F. K., Leakey R. R. B., Ajayi O., Sileshi G., Tchoundjeu Z., Matakala P., et al. (eds). Indigenous fruit trees in the tropics: Domestication, utilization and commercialization. Oxford, United Kingdom, CABI Publishing, 186-203. https://www.cabi.org/cabebooks/ ebook/20083134907

Kokou K., Sokpon N., 2006. Les forêts sacrées du couloir de Dahomey. Bois et Forêts des Tropiques, 288 (2) : 15-23. https:// revues.cirad.fr/index.php/BFT/article/view/20312

Maxted N., Scholten M. A., Codd R., Ford-Lloyd B. V., 2007. Creation and use of a national inventory of crop wild relatives. Biology Conservation, 140 (1-2): 142-159. https://doi.org/10.1016/j. biocon.2007.08.006

Nolan J. M., Turner N. J., 2011. Ethnobotany: The study of peopleplant relationships. In: Anderson E. N., Pearsall D., Hunn E., Turner N. (eds). Ethnobiology. Hoboken, NJ, USA, Wiley-Blackwell, 133-147. https://doi.org/10.1002/9781118015872.ch9

Onoagbe I. O., Attah V., Luther M. M., Esekheigbe A., 1999. Hypoglycemica and anti-diabetic effects of Morinda lucida and Tetracera alnifolia in normal and streptozotocin-induced diabetic rabbits, West Africa. Journal of Biology Science, 9: 1-8.

Palla F., Louppe D., 2002. Obeché. Cirad, France, 6 p. https://agritrop.cirad.fr/515653/1/document 515653.pdf

Pilgrim S. E., Cullen L. C., Smith D. J., Pretty J., 2008. Ecological knowledge is lost in wealthier communities and countries. Environmental Science \& Technology, 42 (4): 1004-1009. https://doi. org/10.1021/es070837v

Quiroz D., van Andel T., 2015. Evidence of a link between taboos and sacrifices and resource scarcity of ritual plants. Journal of Ethnobiology and Ethnomedicine, 11: 5. https://doi. org/10.1186/1746-4269-11-5

Rajesh B., Nair N., 2018. Ethnobotanical studies of medicinal plants used in traditional (folk) medicines. International Journal of Science and Research, 8 (3): 437-442.

Saynez-Vaquez A., Vibrans H., Vergara-Silva F., Caballero J., 2016. Intracultural differences in local botanical knowledge and knowledge loss among the Mexican Isthmus Zapotecs. PLoS ONE, 11 (3): e0151693. https://doi.org/10.1371/journal. pone. 0151693

Siepel A., Poorter L., Hawthorne W., 2004. Ecological profiles of large timber species. In: Poorter L., Bongers F., Kouame F. N., Hawthorne W. D. (eds). Biodiversity of West African Forests. An Ecological Atlas of Woody Plant Species. Oxon, United Kingdom, CABI, 391-446.

Souto T., Ticktin T., 2012. Understanding interrelation ships among predictors (age, gender, and origin) of local ecological knowledge. Economic Botany, 66 (2): 149-164. https://doi. org/10.1007/s12231-012-9194-3

Sundriyal M., Sundriyal R. C., Sharma E., 2004. Dietary use of wild resources in the Sikkim Himalaya, India. Economic Botany, 58 (4): 626-638. https://www.jstor.org/stable/4256875

TRAMIL, 1989. Vers une Pharmacopée Caraïbe (TRAMIL4). Tegucilpa, Honduras, enda-caribe, Université nationale autonome du Honduras (UNAH), 475 p.
Voeks R. A., 2007. Are women reservoirs of traditional plant knowledge? Gender, ethnobotany and globalization in northeast Brazil. Singapore Journal of Tropical Geography, 28: 7-20. https://lib.icimod.org/record/9107

\section{Financement}

Cette étude a été financée grâce à une bourse doctorale octroyée à G. Ganka par le Gouvernement du Bénin et un soutien additionnel du troisième auteur A.B. Fandohan.

\section{Condition d'accès aux données}

Les données ont été obtenues par enquêtes ethnobotaniques auprès des acteurs des forêts sacrées du culte « Oro » et des autres divinités liées à la conservation de Triplichiton scleroxylon K. Schum. au Bénin en respect du code d'éthique établi par la Société internationale d'ethnobiologie (ISE, 2006). Les données sont accessibles avec le lien internet suivant et en sollicitant l'accord des auteurs : https://www.scidb.cn/s/qlvIVz

https://doi.org/10.11922/sciencedb.01491

\begin{tabular}{|c|c|}
\hline \multicolumn{2}{|c|}{ Ganka et al. - Author's contributions } \\
\hline Rôle du contributeur & Noms des auteurs \\
\hline Conceptualisation & G. Ganka, A. B. Fandohan \\
\hline Gestion des données & G. Ganka, A. B. Fandohan \\
\hline Analyse formelle & G. Ganka, A. B. Fandohan, K. V. Salako \\
\hline $\begin{array}{l}\text { Acquisition } \\
\text { du financement }\end{array}$ & G. Ganka, A. B. Fandohan \\
\hline $\begin{array}{l}\text { Enquête } \\
\text { et investigation }\end{array}$ & G. Ganka \\
\hline Méthodologie & G. Ganka, A. B. Fandohan, K. V. Salako \\
\hline Gestion de projet & G. Ganka, A. B. Fandohan \\
\hline Ressources & G. Ganka, A. B. Fandohan \\
\hline Logiciels & G. Ganka, A. B. Fandohan, K. V. Salako \\
\hline Supervision & A. B. Fandohan \\
\hline Visualisation & G. Ganka \\
\hline $\begin{array}{l}\text { Écriture - Préparation } \\
\text { de l'ébauche originale }\end{array}$ & G. Ganka, A. B. Fandohan \\
\hline $\begin{array}{l}\text { Écriture - Révision } \\
\text { et édition }\end{array}$ & G. Ganka, A. B. Fandohan, K. V. Salako \\
\hline
\end{tabular}

Bois et Forêts des Tropiques - Revue scientifique du Cirad (c) Bois et Forêts des Tropiques () Cirad
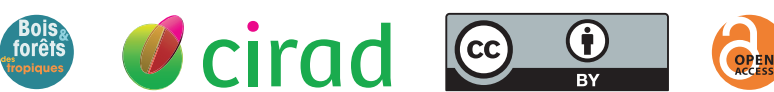

Cirad - Campus international de Baillarguet,

34398 Montpellier Cedex 5, France

Contact : bft@cirad.fr - ISSN : L-0006-579X 